\title{
Tagging and Chunking with Bigrams
}

\author{
Ferran Pla, Antonio Molina and Natividad Prieto \\ Universitat Politècnica de València \\ Departament de Sistemes Informàtics i Computació \\ Camí de Vera s/n \\ 46020 València \\ $\{$ fpla,amolina,nprieto $\} @ d s i c . u p v . e s$
}

\begin{abstract}
In this paper we present an integrated system for tagging and chunking texts from a certain language. The approach is based on stochastic finite-state models that are learnt automatically. This includes bigram models or finite-state automata learnt using grammatical inference techniques. As the models involved in our system are learnt automatically, this is a very flexible and portable system.

In order to show the viability of our approach we present results for tagging and chunking using bigram models on the Wall Strect Journal corpus. We have achicved an accuracy rate for tagging of $96.8 \%$, and a precision rate for NP chunks of $94.6 \%$ with a recall rate of $93.6 \%$.
\end{abstract}

\section{Introduction}

Part of Speech Tagging and Shallow Parsing are two well-known problems in Natural Language Processing. A Tagger can be considered as a translatior that reads sentences from a certain language and outputs the corresponding scquences of part of speech (POS) tags, taking into account the context in which each word of the sentence appears. A Shallow Parser involves dividing sentences into non-overlapping segments on the basis of very superficial analysis. It includes discovering the main constitucnts of the sentences (NPs, VPs, PPs, ...) and their heads. Shallow Parsing usually identifies non-recursive constituents, also called chunks (Abncy, 1991) (such as non-recursive Noun Phrases or base NP, base VP, and so on). It can include determining syntactical relationships such as subject-verb, verb-object, etc., Shallow parsing which always follows the tagging process, is used as a fast and reliable pre-processing phase for full or partial parsing. It can be used for Information Retrieval Systems, Information Extraction, Text Summarization and Bilingual Alignment. In addition, it is also used to solve computational linguistics tasks such as disambiguation problems.

\subsection{POS Tagging Approaches}

The different approaches for solving this problem can be classified into two main classes depending on the tendencies followed for establishing the Language Model (LM): the linguistic approach, based on hand-coded linguistic rules and the learning approach derived from a corpora (labelled or nonlabelled). Other approximations that use hybrid methods have also been proposed (Voutilainen and Padró, 1997).

In the linguistic approach, an expert linguist is needed to formalise the restrictions of the language. This implies a very high cost and it is very depondent on each particular language. We can find an important contribution (Voutilainen, 1995) that uses Constraint Grammar formalism. Supervised learning methods were proposed in (Brill, 1995) to learn a set of transformation rules that repair the error committed by a probabilistic tagger. The main advantage of the linguistic approach is that the model is constructed from a linguistic point of view and contains many and complex kinds of knowledge.

In the learning approach, the most cxtended formalism is based on n-grams or IMMM. In this case, the language model can be estimated from a labelled corpus (supervised methods) (Church, 1988)(Weischedel et al., 1993) or from a nonlabelled corpus (unsupervised methods) (Cutting et al., 1992). In the first case, the model is trained from the relative observed frequencies. In the second one, the model is learned using the Baum-Welch algorithm from an initial model which is estimated using labelled corpora (Merialdo, 1994). The advantages of the unsupervised approach are the facility to build language models, the flexibility of choice of catcgories and the ease of application to other languages. We can find some other machinc-learning approaches that usc more sophisticated LMs, such as Decision Trees (Màrquez and Rodríguez, 1998)(Magerman, 1996), memory-based approaches to learn special decision trees (Daelemans et al., 1996), maximum entropy approaches that combine statistical information from different sources (Ratnaparkhi, 1996), finite state automatia inforred using Grammatical Inference (Pla and Pricto, 1998), ctc.

The comparison among different approaches is difficult due to the multiple factors that can be consid- 
cred: the language, the number and type of the tags, the size of the vocabulary, the ambignity, the difficulty of the test set, etc. 'The best results reported on the Wall Street, Journal (WSJ) Treobank (Marcus et al., 1993), using statistical language models, have an accuracy ratio between $95 \%$ and $97 \%$ (depending on the diflerent factors mentioned above). For the linguistic approach the results ane better. For example, in (Voutilaincn, 1995) an accuracy of $99.7 \%$ is reported, but certain ambiguitios in the output semain unsolved. Somo works have rocently been pulblished (Brill and Wu, 1998) in which a set of taggers are combined in order to improve their performance. In some cases, these methods achicve an accuracy of $97.9 \%$ (Haltoron et al., 1.998).

\subsection{Shallow Parsing $\Lambda$ pproaches}

Since the carly 90 's, scveral techniques for carrying out shallow parsing have been devoloped. 'These? techniques can also be classiliced into two main groups: based on hand-coded linguistic rules and based on learning algorithms. These approaches have a common characteristic: they take the sequence of lexical tags proposed by a POS tagger as input, for both tho learning and the chunking processes.

\subsubsection{Techniques based on hand-coded linguistic: rules}

These methods use a hand-wititen set of rules that are defined using POS as terminals of the grammar. Most of these works use finite state methods for detecting chnnks or for accomplishing other linguistic tasks (Ejcrhed, 1988), (Aloncy, 1996), (AtMokhtar and Chanod, 1997). Other works use different grammatical formalisms, such as constraint grammars (Voutilainen, 1993), or connbine the grammar rules with a set of hemistics (Bouriganlt, 1992). These works usually use a small test set that is manually evaluated, so the achieved results aro not significant. The regular expressions defined in (Ljerhed, 1988) identified both non--ecursive clauses and non-recursive NI's in English text. The experinentation on the Brown corpus achieved a precision rate of $87 \%$ (for clauses) and $97.8 \%$ (for NPs). Al)ney introduced the concept of chunk (A.bney, 1991) and presented an incremential partial parser (Abncy, 1996). This parser identilies chunks base on the parts of spech, and it then chooses how to combine them for higher level analysis using lexical in formation. The average precision and recall rates for chunks were $87.9 \%$ and $87.1 \%$, respectively, on a test set of 1000 sentences. An inciemental architecture of finite-state transducess for French is presented in (At,-Mokhtar and Chanod, 1997). Each transducer performs a linguistic task such as identifying segments or syntactic structures and detecting subjects and objects. The system was evaluated on various corpora for subject and object detection. The precision rate varied betwen $99.2 \%$ and $92.6 \%$. The recall rate varied between $97.8 \%$ and $82.6 \%$.

The NPTool parser described in (Voutilainen, 1993) identified maximal-length noun phrases. NI'tool gave a procision late of $95-98 \%$ and a rocall rate of $98.5-100 \%$. These results were criticised in (Ramshaw and Marcus, 1995) due to some inconsistencies and apparent mistakes which appeared on the sample given in (Voutilainen, 1993). Bourigault developed the LECIER parser for French using grammatical rules and some heuristics (Bourigault, 1992). It achicved a recall rate of $95 \%$ identifying maximal length teminological noun phrases, but he did not give a precision rate, so it is difficult, to evaluate the actual performance of the parser.

\subsubsection{Learning Techniques}

These approaches antomatically construct a language model from a labelled and bracketed corpus. The first probabilistic approach was proposed in (Church, 1988). This mothod loant a bigram model for detecting simple noun phrases on the Brown corpus. Given a sequence of parts of speech as input, the Church program inserts the most probable openings and endings of NPs, using a Viterbi-like dynamic; programming algorithm. Church did not give precision and recall rates. Ho showed that 5 out of 243 NP were omitted, but in a very small test with a POS tagging accuracy of $99.5 \%$.

Transformation-based learning (T'BI) was ned in (Ramshaw and Marcus, 1995) to detect baso Nl? In this work chunking was consirlered as a tagging technique, so that each P'OS could be tagged with 1 (inside baseNP), O (ontsicle basoND) or 13 (inside a baseNP, but the proceding word was in anothes: baseNP). This approach resulted in a precision rate of $91.8 \%$ and a recall rate of $92.3 \%$. This result; was automatically cvaluated on a test set $(200,000$ words) extracted from the WS.J Trecbank. The main drawback to this approach are the high requirements for tine and space which are needed to train the system; it needs to train 100 templates of combinations of words.

There are several works that use a momory-based learning algorithn. These approachos construct a classifier for a task by storing a set of examples in memory. Each cxample is defined by a set of features that lave to be learnt from a bracketed corpus. The Momory-Based Learning (MBJ) algorithm (Daelcmans of al., 1999) takes into account lexical and POS information. It stores the following foatures: the word form and POS tag of the two words to the left, the focus word and one word to the right. This system achieved a precision rate of $93.7 \%$ and a recall rate of $94.0 \%$ on the WS.J Treobank. However, when only P'OS information was used the performance decreased achicving a precision rate of $90.3 \%$ and a 
recall rate of $90.1 \%$. The Memory-Based Sequence Learning (MBSL) algorithm (Argamon et al., 1998) learns substrings or sequences of POS and brackets. Precision and recall rates were $92.4 \%$ on the same data used in (Ramshaw and Marcus, 1995).

A simple approach is presented in (Cardic and Picrce, 1998) called Trecbank Approach (TA). This technique matches $P O S$ sequences from an initial noun phrase grammar which was extracted from an annotated corpus. The precision achieved for each rule is used to rank and prune the rules, discarding those rules whose score is lower than a predefined threshold. It uses a longest match heuristic to dotermine base NP. Precision and recall on the WSJ Trecbank was $89.4 \%$ and $90.0 \%$, respectively.

It is difficult to compare the different approaches due for various reasons. Each onc uses a different definition of basc NP. Each one is evaluated on a different corpus or on different, parts of the same corpus. Some systems have even been evaluated by hand on a very small test set. Table 1 summarizes the precision and recall rates for learning approaches that usc data extracted from the WSJ Treebank.

\begin{tabular}{|c|c|c|}
\hline Method & NP-Precision & NP-Recall \\
\hline TBL & 91.8 & 92.3 \\
\hline MBSL & 92.4 & 92.4 \\
\hline TA & 89.4 & 90.9 \\
\hline MBL & 93.7 & 94.0 \\
\hline MBI (only POS) & 90.3 & 90.1 \\
\hline
\end{tabular}

Table 1: Precision and recall rates for different NP parsers.

\section{General Description of our Integrated approach to Tagging and Chunking}

We propose an integrated system (Figure 1) that combines different knowledge sources (lexical probabilities, LM for chunks and Contextual LM for the sentences) in order to obtain the corresponding sequence of POS tags and the shallow parsing $\left(\left[S U W_{1} / C_{1} W_{2} / C_{2} S U\right] W_{3} / C_{3} \ldots\left[S U W_{n} / C_{n} S U\right]\right)$ from a certain input string $\left(W_{1}, W_{2}, \ldots, W_{n}\right)$. Our system is a transducer composed by two levels: the upper one represents the Contextual LM for the sentences, and the lower one modelize the chunks considered. The formalism that we have used in all levels are finite-state automata. To be cxact, we have used models of bigrams which are smoothed using the backoff technique (Katz, 1987) in order to achieve full coverage of the language. The bigrams LMs (bigram probabilities) was obtained by means of the SLM TOOLKIT (Clarksond and Ronsenfeld,

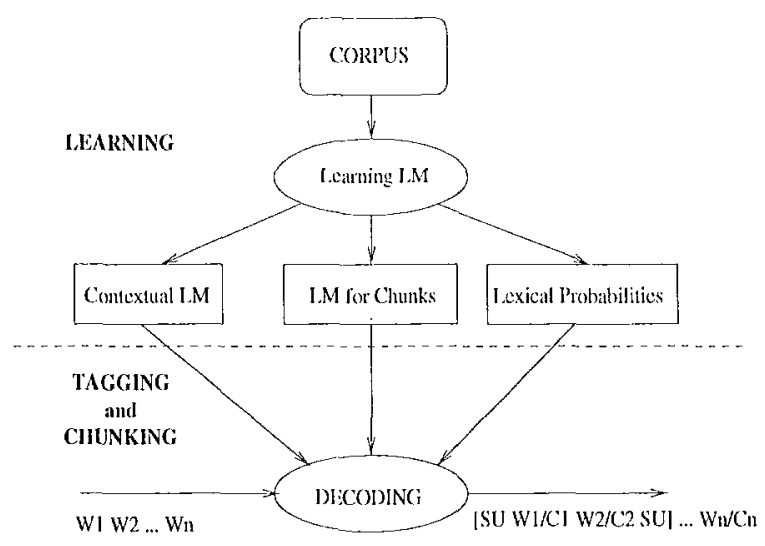

Figure 1: Overview of the System.

1997) from the sequences of categories in the training set. Then, they have been represented like finitc-state automata.

\subsection{The learning phase.}

The models have been estimated from labelled and bracketed corpora. The training set is composed by sentences like:

$\left[S U W_{1} / C_{1} W_{2} / C_{2} S U\right] W_{3} / C_{3} \ldots\left[S U W_{n} / C_{n} S U\right] . /$

where $W_{i}$ are the words, $C_{i}$ are part-of-speech tags and $\mathrm{SU}$ are the chunks considered.

The modols learnt arc:

- Contextual LM: it is a smoothed bigram model learnt from the sequences of part-of-specch tags $\left(C_{i}\right)$ and chunk descriptors $(S U)$ present in the training corpus (sce Figure $2 \mathrm{a}$ ).

- Models for the chunks: they are smoothod bigram models learnt from the sequences of partof-specch tags corresponding to cach chunk of the training corpus (sce Figure 2b).

- Lexical Probabilities: they are estimated from the word frequencies, the tag frequencies and the word per tag frequencies. A tag dictionary is used which is built from the full corpus which gives us the possible lexical categories (POS tags) for cach word; this is equivalent to having an ideal morphological analyzer. The probabilities for each possible tag are assigned from this information taking into account the obtained statistics. Due to the fact that the word cannot have been seen at training, or it has only been seen in some of the possible categories, it is compulsory to apply a smoothing mechanism. In our case, if the word has not previously been seen, the samo probability is assigned to all the categories given by the dictionary; if it has been seen, but not in all the 


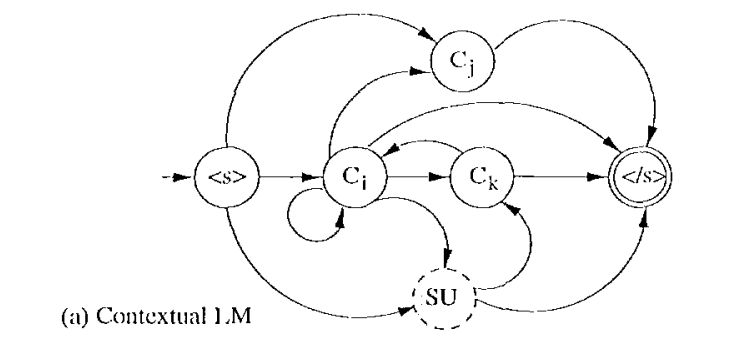

(b) I.M for Chunks

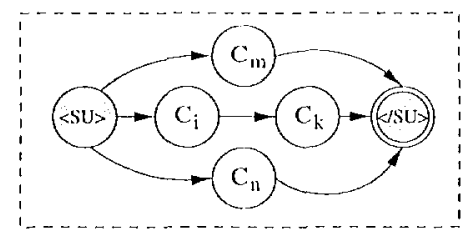

(c) Integrated LM

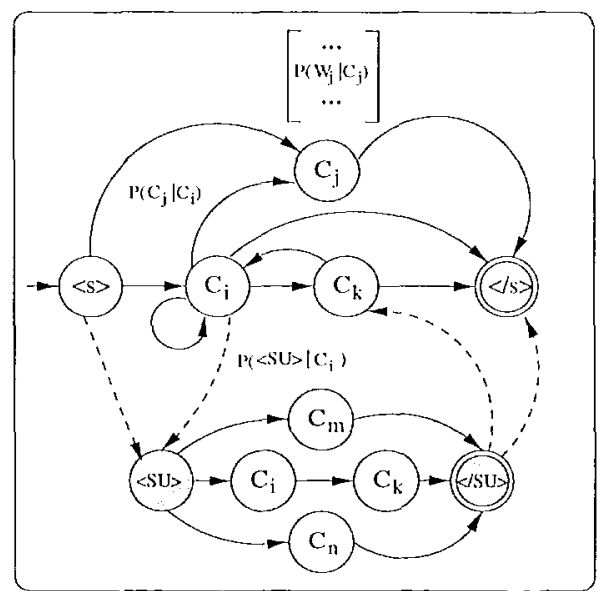

Figure 2: Integrated Ianguage Model for Tagging and Chunking.

categories, the smoothing called "add one" is applied. Afterwards, a renormalization process is carried out.

Once the LMs have been learnt, a regular substitution of the lower model(s) into the upper one is made. In this way, wo get a single Integrated LM which shows the possible concatenations of lexical tags and syntactical units, with their own transition probabilities which also include the lexical probabilities as well (seo Figure 2c). Note that the models in Figure 2 are not smoothed).

\subsection{The Decoding Process: Tagging and Parsing}

The tagging and shallow parsing process consists of finding out the sequence of states of maximum probability on the Integrated LM for an input sentence. Therefore, this sequence must be compatible with the contextual, syntactical and lexical constraints. This process can be carried out by Dynamic Programming using the Viterbi algorithm, which is conveniently modified to allow for transitions between certain states of the automata without consuming any symbols (epsilon transitions). A portion of the Dynamic Programming trellis for a generic sentence using the Integrated LM shown in Figure 2c can be seen in Figure 3. The states of the automata that can be reached and that are compatible with the lexical constraints are marked with a black circle (i.c., from the state $C_{k}$ it is possible to reach the state $C_{i}$ if the transition is in the automata and the lexical probability $P\left(W_{i} \mid C_{i}\right)$ is not null). Also, the transitions to initial and final states of the models for chunks (i.e., from $C_{i}$ to $\langle S U>$ ) are allowed; these states are marked in Figure 3 with a white circle and in this case no symbol is consumed. In all these cases, the transitions to initial and final pro- duce transitions to their successors (the dotted lines in ligure 3) where now symbols must be consumed.

Once the Dynamic Programing trellis is built, we can obtain the maximum probability path for the input sentence, and thus the best sequence of lexical tags and the best segmentation in chunks.

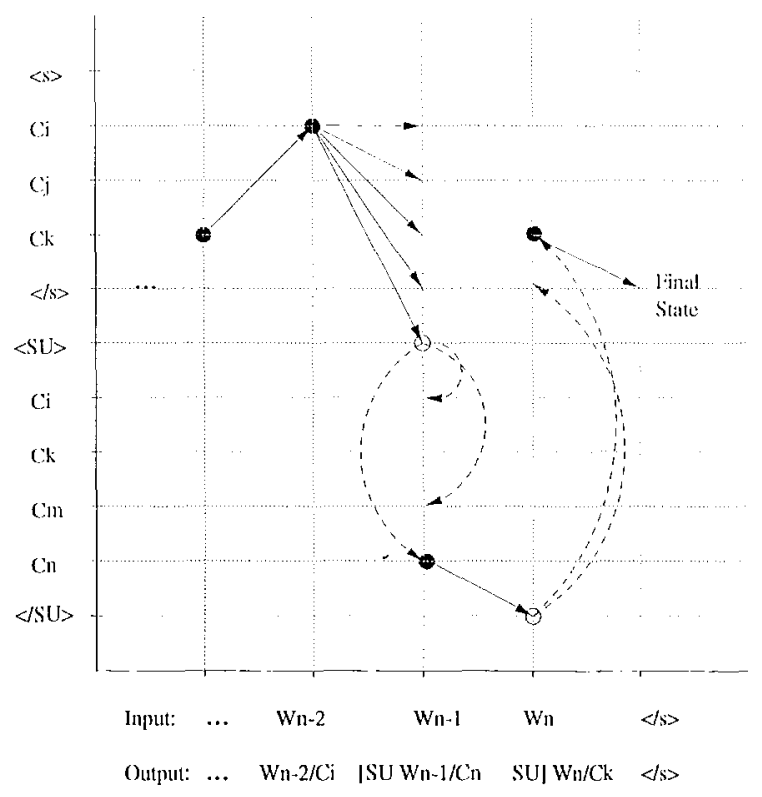

Figure 3: Partial Trellis for Programming Decoding based on the Integrated LM.

\section{Experimental Work}

In this section we will describc a set of experiments that we carried out in order to demonstrate the capabilities of the proposed approach for tagging and shallow parsing. The experiments were carried out 
on the WSJ corpus, using the POS tag set defined in (Marcus et al., 1993), considering only the NP chunks defined by (Church, 1988) and using the models that we have presented above. Nevertheless, the use of this approach on other corpora (changing the reference language), other lexical tag scts or other kinds of chunks can be done in a direct way.

\subsection{Corpus Description.}

We used a portion of the WSJ corpus $(900,000$ words), which was tagged according to the Penn Treebank tag set and bracketed with NP markers, to train and test the system.

The tag set contained 45 different tags. About $36.5 \%$ of the words in the corpus were ambiguous, with an ambiguity ratio of $2.44 \mathrm{tag} /$ word over the ambiguous words, 1.52 overall.

\subsection{Experimental Results.}

In order to train the models and to test the system, we randomly divided the corpora into two parts: approximately 800,000 words for training and 100,000 words for testing.

Both the bigram models for representing contextual information and syntactic description of the NP chunk and the lexical probabilities were estimated from training sets of different sizes. Due to the fact that we did not use a morphological analyser for English, we constructed a tag dictionary with the lexicon of the training set and the test set used. This dictionary gave us the possible lexical tags for cach word from the corpus. In no case, was the test used to estimate the lexical probabilities.

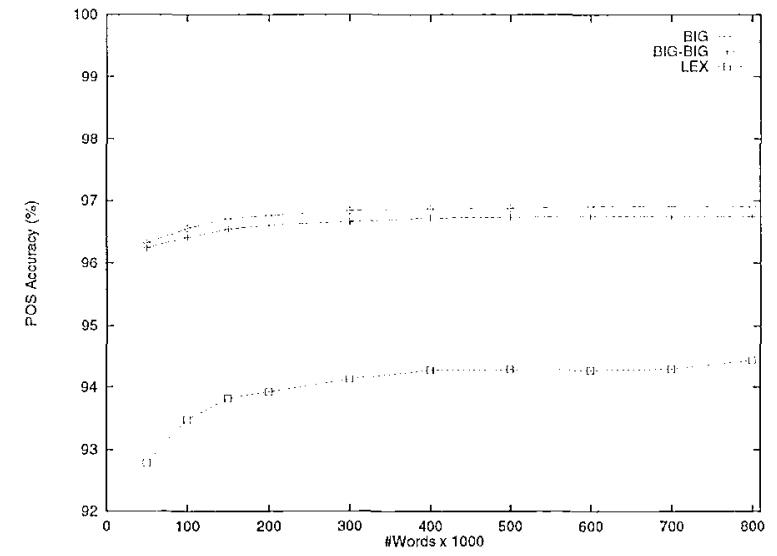

Figure 4: Accuracy Rate of Tagging on WSJ for incromental training sets.

In Figure 4, we show the results of tagging on the test set in terms of the training set size using thrce approaches: the simplest (LEX) is a tagging process which does not take contextual information into account, so the lexical tag associated to a word will

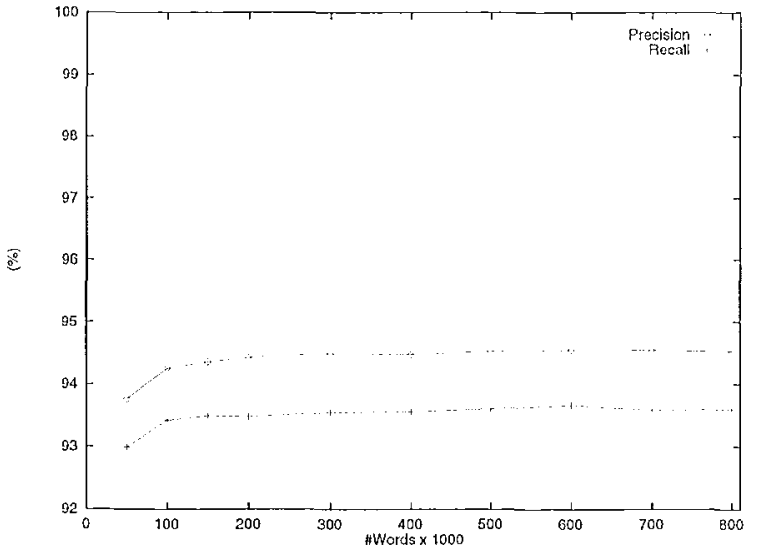

Figure 5: NP-chunking results on WSJ for incremental training sets.

\begin{tabular}{|l|l|l|l|}
\cline { 2 - 4 } \multicolumn{1}{c|}{} & \multicolumn{1}{c|}{ Tagging } & \multicolumn{2}{c|}{ NP-Chunking } \\
\hline Tagger & \multicolumn{1}{c|}{ Accuracy } & Precision & Recall \\
\hline \hline BIG-BIG & 96.8 & 94.6 & 93.6 \\
\hline \hline Lex & 94.3 & 90.8 & 91.3 \\
\hline BIG & 96.9 & 94.9 & 94.1 \\
\hline IDEAL & 100 (assumed) & 95.5 & 94.7 \\
\hline
\end{tabular}

Table 2: Tagging and NP-Chunking results for differents taggers (training set of 800,000 words).

be that which has appeared more often in the training set. The second method corresponds to a tagger based on a bigram model (BIG). The third onc uses the Integrated LM described in this paper (BIGBIG). The tagging accuracy for BIG and BIG-BIG was close, $96.9 \%$ and $96.8 \%$ respectively, whereas without the use of the language model (LEX), the tagging accuracy was 2.5 points lower. The trend in all the cases was that an increment in the size of the training set resulted in an increase in the tagging accuracy. After 300,000 training words, the result became stabilized.

In Figure 5, we show the precision (\#correct proposed NP/\#proposed NP) and recall (\#correct proposed $\mathrm{NP} / \# \mathrm{NP}$ in the reference) rates for $\mathrm{NP}$ chunking. The results obtained using the Integrated LM were very satisfactory achieving a precision rate of $94.6 \%$ and a recall rate of $93.6 \%$. The performance of the NP chunker improves as the training set size increases. This is obviously due to the fact that the model is better learnt when the size of the training set increases, and the tagging error: decreases as we have secn above.

The usual sequential process for chunking a sentence can also be used. That is, first we tag the sentence and thon we use the Integrated LM to carry out the chunking. In this case, only the contextual probabilities are taken into account in the decoding 
process. In Table 2, wo show the most relevant results that wo obtained for tagging and for NP chunking. The first row shows the result when the tagging and the chunking are done in a integrated way. The following rows show the performance of the sequential process using differcnt taggers:

- IJXX: it takes into account only lexical probabilities. In this case, the tagging accuracy was $94.3 \%$.

- BIG: it is based on a bigram model that achieved an accuracy of $96.9 \%$.

- IDEAL: it simulates a tagger with an accuracy rate of $1.00 \%$. To do this, we used the tagged sentences of the WSJ corpus directly.

These results confirm that precision and recall rates increase when the accuracy of the tagger is better. The performance of the sequential process (using the BIG tagger) is slightly better than the performance of the integrated process (BIG-BIG). We think that this is probably bocause of the way we combined the probabilities of the different models.

\section{Conclusions and Future Work}

In this paper, we have presented a system for Tagging and Chunking based on an Integrated language Model that uses a homogeneous formalism (finite-state machine) to combine different knowledge sourcos: lexical, syntactical and contextnal models. It is feasible both in torns of performance and also in terms of computational efliciency.

All the models involved arc learnt automatically from data, so the system is very flexible and portable and changes in the reference language, lexical tags or other kinds of chunks can be made in a direct way.

The tagging accuracy $(96.9 \%$ using BIG and 96.8\% using BIG-BIG) is higher than other similar approaches. 'This is because we have used the tag dictionary (including the test set in it) to restrict the possible tags for unknown words, this assumption obviously increase the rates of tagging (we have not done a quantitative study of this factor).

As we have mentioned above, the comparison with other approaches is difficult duc among other reasons to the following ones: the definitions of base NP are not always the same, the sizes of the train and the test sets are different and the knowledge sources used in the learning process are also different. The precision for NP-chunking is similar to other statistical approaches presented in section 1 , for both the integrated process (94.6\%) and the sequential process using a tagger based on bigrams (94.9\%). The rocall rate is slightly lower than for some approaches using the integrated system (93.6\%) and is similar for the sequential process (94.1\%). When wo used the soquential system taking an error free input (IDEAL), the performance of the system obviously increased (95.5\% precision and $94.7 \%$ recall). These results show the influcnce of tagging errors on the process. Nevertheless, we are studying why the results botween the integrated process and the sequential process are different. We are testing how the introduction of some adjustment factors among the models for weighting the different probability distribution can improve the results.

The models that we have used in this work, are bigrams, but trigrams or any stochastic regular model can be used. In this respect, we have worked on a more complex LMs, formalized as a finite-state automata which is learnt using Grammatical Inference techniques. Also, our approach would benefit from the inclusion of loxical-contextual information into the LM.

\section{Acknowledgments}

This work has becn partially supported by the Spanish Research Project, CICYT (TIC97-0671-C02$01 . / 02)$.

\section{References}

S. Abney. 1991. Parsing by Chunks. R. Berwick, S. Abncy and C. 'Tonny (cds.) Principle-based Parsing. Kluwer Academic Publishers, Dordrecht.

S. Abney. 1996. Partial Parsing via Finite-State Cascades. In Procecdings of the ESSLLI'96 Robust Parsing Workshop, Prague, Cyech Republic.

S. Arganon, I. Dagan, and Y. Krymolowski. 1998. A Memory based Approach to Leaming Sliallow Natural Language Patterns. In Proceedings of the joint 17th International Conference on Computational Linguistics and 36th Annual Mecting of the Association for Computational Linguistics, COLING-ACL, pages 67-73, Montréal, Canada.

S. At-Mokhtar and J.P. Chanod. 1997. Incremental Finite-State Parsing. In Procedings of the 5th Conference on Applied Natural Ianguage Process$i n g$, Washington D.C., USA.

D. Bourigault. 1992. Surface Grammatical Analysis for the Fxtraction of Terminological Noum Phrases. In Procedings of the 15th International Confercnce on Computational Linguistics, pages 977-981.

Eric Brill and Jun Wu. 1998. Classificr Combination for Improved Lexical Disambiguation. In Procedings of the joint 17 th International Conference on Computational Linguistics and 36th Annual Meeting of the Association for Computational Linguistics, COLING-ACI, pages 191-195, Montréal, Canada.

E. Brill. 1995. 'Transformation-based Error--clriven Loarning and Natural Language Processing: A 
Case Study in Part-of-speech Tagging. Computational Linguistics, 21(4):543-565.

C. Cardie and D. Pierce. 1998. Error-Driven Prunning of Treebank Grammars for Base Noun Pliraso Identification. In Proceedings of the joint 17th International Conference on Computational Linguistics and 36th Annual Mecting of the Association for Computational Linguistics, COLING$A C L$, pages 218-224, Montréal, Canada, August.

K. W. Church. 1988. A Stochastic Parts Program and Noun Phrase Parser for Unrestricted Text. In Proceedings of the 1st Conference on Applied Natural Language Processing, ANLP, pages 136143. ACL.

P. Clarksond and R. Ronsenfeld. 1997. Statistical Language Modeling using the CMU-Cambridge Toolkit. In Proceedings of Eurospecch, Rhodes, Greece.

D. Cutting, J. Kupicc, J. Pederson, and P. Sibun. 1992. A Practical Part-of-speech Tagger. In Proceedings of the 3rd Conference on Applied Natural Language Processing, ANLP, pages 133-140. ACL.

W. Daelemans, J. Zavrel, P. Berck, and S. Gillis. 1996. MBT: A Memory-Based Part-of-speech Tagger Generator. In Proceedings of the 4 th Workshop on Very Large Corpora, pages 14-27, Copenhagen, Denmark.

W. Daelemans, S. Buchholz, and J. Veenstra. 1999. Memory-Based Shallow Parsing. In Proceedings of $E M N L P / V L C-99$, pages 239-246, University of Maryland, USA, June.

E. Ejcrhed. 1988. Finding Clauses in Unrestricted Text by Finitary and Stochastic Methods. In Proceedings of Second Conference on Applied Natural Language Processing, pages 219-227. ACL.

H. van Halteren, J. Zavrel, and W. Daclemans. 1998. Improving Data Driven Wordclass Tagging by System Combination. In Proceedings of the joint 17th International Conference on Computational Linguistics and 36th Annual Meeting of the Association for Computational Linguistics, COLING$A C L$, pages 491-497, Montréal, Canada, August.

S. M. Katz. 1987. Estimation of Probabilities from Sparse Data for the Language Model Component of a Speech Recognizer. IEEE Transactions on Acoustics, Speech and Signal Processing, 35.

D. M. Magerman. 1996. Learning Grammatical Structure Using Statistical Decision-Trees. In Proceedings of the 3rd International Colloquium on Grammatical Inference, ICGI, pages 1-21. Springer-Verlag Lecture Notes Series in Artificial Intelligence 1147.

M. P. Marcus, M. A. Marcinkiewic $z$, and B. Santorini. 1993. Building a Large Annotated Corpus of English: The Penn Treebank. Computational Linguistics, $19(2)$.
Lluís Màrquez and Horacio Rodríguez. 1998. Partof-Speech Tagging Using Decision Trees. In C. Nédellec and C. Rouveirol, editor, LNAI 1398: Proceedings of the 10th European Conference on Machine Learning, ECML'98, pages 25-36, Chemnitz, Germany. Springer.

B. Mcrialdo. 1994. Tagging English Text with a Probabilistic Model. Computational Linguistics, 20(2):155-171.

F. Pla and N. Prieto. 1998. Using Grammatical Inference Methods for Automatic Part-of-spech Tagging. In Proceedings of 1st International Conference on Language Resources and Evaluation, LREC, Granada, Spain.

L. Ramshaw and M. Marcus. 1995. Text Chunking Using Transformation-Based Learning. In Procecdings of third Workshop on Very Large Corpora, pages $82-94$, June.

A. Ratnaparkhi. 1996. A Maximum Entropy Partof-speech Tagger. In Proceedings of the 1st Conference on Empirical Methods in Natural Language Processing, EMNISP.

Atro Voutilainen and Lluís Padró. 1997. Developing a Hybrid NP Parser. In Proceedings of the 5th Conference on Applied Natural Language Processing, ANLP, pages 80-87, Washington DC. ACL.

Atro Voutilainen. 1993. NPTool, a Detector of English Noun Phrases. In Proceedings of the Workshop on Very Large Corpora. ACL, June.

Atro Voutilainen. 1995. A Syntax-Based Part-ofspecch Analyzer. In Proceedings of the 7th Conference of the European Chapter of the Association for Computational Linguistics, EACL, Dublin, Ireland.

R. Woischedel, R. Schwart\%, J. Palmucci, M. Metecr, and L. Ramshaw. 1993. Coping with Ambiguity and Unknown Words through Probabilistic Models. Computational Linguistics, 19(2):260-269. 